\title{
EQUIVALENCE OF CERTAIN CATEGORIES OF MODULES FOR QUANTIZED AFFINE LIE ALGEBRAS
}

\author{
VYACHESLAV M. FUTORNY and DUNCAN J. MELVILLE
}

(Received 7 April 1999; revised 28 February 2000)

Communicated by C. F. Miller

\begin{abstract}
We show that a quantum Verma-type module for a quantum group associated to an affine Kac-Moody algebra is characterized by its subspace of finite-dimensional weight spaces. In order to do this we prove an explicit equivalence of categories between a certain category containing the quantum Verma modules and a category of modules for a subalgebra of the quantum group for which the finite part of the Verma module is itself a module.
\end{abstract}

2000 Mathematics subject classification: primary 17B37, 17B67, 81R50.

\section{Introduction}

In this paper, we determine an equivalence of categories between certain representations of the quantum group and some representations of a particular subalgebra. The main purpose of constructing this category equivalence is to establish an equivalence between quantum Verma-type modules and modules that are essentially their finite parts. In the classical case, that is representations of affine Kac-Moody algebras, analogous results were obtained in [CFM]. However, in the quantum case, the techniques required and, indeed, the precise statements of the results differ somewhat from those in the classical case.

Verma-type modules for affine Kac-Moody algebras are constructed in a similar fashion to ordinary Verma modules except that they are induced from non-standard

Research of the first author was partially supported by the CRDF grant. Regular Associate of the ICTP. Research of the second author was partially supported by a Faculty Research Grant from St. Lawrence University.

(c) 2000 Australian Mathematical Society 0263-6115/2000\$A2.00+0.00 
Borel subalgebras (that is, those not Weyl-equivalent to standard Borel subalgebras). Verma-type modules were originally introduced and classified by Jakobsen and Kac [JK1, JK2] and independently in [Fu1, Fu2]. They are in many ways similar to ordinary Verma modules, but they typically have both finite and infinite-dimensional weight spaces. However, as long as the central element in the affine algebra acts by a non-zero charge, the structure of these Verma-type modules is essentially determined by the subspace of finite-dimensional weight spaces. This subspace is, of course, not a submodule for the whole affine algebra, but one can construct a suitable subalgebra for which the finite part of the Verma-type module is a module. One of the main results of [CFM] was establishing the equivalence of these representations.

The construction and analysis of quantum Verma-type modules is hampered somewhat by the lack of a general PBW theorem for quantum groups. This means one has to be very cautious about subalgebras constructed in terms of generators and relationsthe commutation relations for arbitrary elements of a quantum group are not well understood. The case of quantum Verma-type modules for $U_{q}\left(A_{1}^{(1)}\right)$ was considered in [CFKM], where it was shown that these modules were true quantum deformations of Verma-type modules for the underlying affine algebra. The techniques used in [CFKM] do not easily generalize to other affine algebras but, by utilizing a rather different approach, the same quantum deformation results were obtained in [FGM]. That paper also contains several structural results on imaginary Verma modules (a subclass of Verma-type modules).

Here we build upon, and make many references to, the results of [FGM]. The most important insight in [FGM] was that, while one may be unsure about a basis for a subalgebra of $U_{q}(\mathfrak{g})$ determined by certain generators, one may still be able to determine a basis for representations generated by that subalgebra. This result [FGM, Theorem 3.5] is quoted below, used repeatedly, and the proof of our crucial Proposition 6.1 below uses similar arguments to the proof of [FGM, Theorem 3.5]. The construction of a basis for our representations depends in turn on having detailed information about an explicit basis for the quantum group and for this we draw heavily on the work of Beck and Kac on PBW bases of quantum groups [BK]. We quote the necessary machinery below.

Although our principal concern here is with quantum modules, in order to establish certain results, we do at times need to refer to previously obtained classical results. The connection between the quantum and classical cases is via the $\mathbb{A}$-form approach to quantum deformations originally due to Lusztig. We do not provide complete details of this technique here, but refer the reader to [FGM], where this process is given in full.

In Section 2, we establish notation and recall results and constructions from [BK] and [FGM]. Section 3 constructs representations of the subalgebra $\mathfrak{g}^{J}$ of $U_{q}(\mathfrak{g})$. Section 4 discusses representations of quantum Heisenberg algebras and refers to 
structural results obtained in [Fu3] in the non-quantum case. In Section 5 we introduce the category $\mathscr{O}^{J}(\lambda)$ of $\mathfrak{p}^{J}$-modules that contains the finite parts of the Verma-type modules. Finally, Section 6 establishes the equivalence between the category $\mathscr{O}^{J}(\lambda)$ and a category $\mathscr{O}_{J}(\lambda)$ of $U_{q}(\mathfrak{g})$-modules containing the Verma-type modules.

Much of the research reported in this paper was conducted while the authors were visiting the University of Wisconsin, Madison in January 1999. We would like to thank the University of Wisconsin, and Georgia Benkart in particular, for their very gracious hospitality during this visit.

\section{Preliminaries}

We introduce some required notation and terminology. For more details of the basic constructions, the reader is referred to [FGM]. We work with a particular basis of the quantum group, and a parametrization of the closed partition of the root system, so we introduce these topics first.

Let $\mathfrak{g}$ be an (untwisted) affine Kac-Moody algebra with root system $\Delta$ and set of simple roots $\Pi$. Then

$$
\Delta=\{\alpha+n \delta \mid \alpha \in \dot{\Delta}, n \in \mathbb{Z}\} \cup\{k \delta \mid k \in \mathbb{Z}, k \neq 0\},
$$

where $\dot{\Delta}$ is the root system for the underlying finite-dimensional simple algebra $\dot{\mathfrak{g}}$ and $\delta$ is the minimal positive imaginary root of $\mathfrak{g}$. We let $\Delta_{ \pm}$denote the sets of positive and negative roots, $Q$ the root lattice and $Q_{ \pm}$the monoids generated by $\Delta_{ \pm}$.

Following Beck and Kac ([BK], see also $[\mathrm{KT}])$, we introduce a total ordering of positive roots of $\mathfrak{g}$

$$
\beta_{0}>\beta_{-1}>\beta_{-2}>\cdots>\delta>2 \delta>\cdots>\beta_{2}>\beta_{1},
$$

where $\left\{\beta_{k} \mid k \leq 0\right\}=\left\{\alpha+n \delta \mid \alpha \in \dot{\Delta}_{+}, n \geq 0\right\}$ and $\left\{\beta_{k} \mid k \geq 1\right\}=\{-\alpha+n \delta \mid \alpha \epsilon$ $\dot{\Delta}^{+}, n>0$ ). Clearly, by declaring $-\alpha<-\beta$ if and only if $\beta>\alpha$ for the positive roots $\alpha, \beta$ we obtain a corresponding ordering on the negative roots. All negative roots are considered smaller than positive roots.

Let $I=\{0,1, \ldots, N\}$ be a set indexing the simple roots of $\mathfrak{g}$ so that $\Pi=\left\{\alpha_{i} \mid\right.$ $i \in I\}$. We let $A=\left(a_{i j}\right)_{0 \leq i, j \leq N}$ be the Cartan matrix of $\mathfrak{g}$. Let $\mathfrak{h}$ denote the Cartan subalgebra of $\mathfrak{g}$ with $h_{i} \in \mathfrak{h}$ defined by $\alpha_{i}\left(h_{j}\right)=a_{i j}$ for $i, j \in I$. We also let $c$ denote the canonical central element of $\mathfrak{g}$ and $d$ the degree derivation. The weight lattice of $\mathfrak{g}$ is $P=\left\{\lambda \in \mathfrak{h}^{*} \mid \lambda\left(h_{i}\right) \in \mathbb{Z}, i \in I, \lambda(d) \in \mathbb{Z}\right\}$. For more details see [FGM].

The quantum group, $U_{q}(\mathfrak{g})$, of $\mathfrak{g}$ is an associative algebra with 1 over $\mathbb{C}(q)$ with generators $E_{i}, F_{i}, K_{i}^{ \pm 1}(i \in I)$ and $D^{ \pm 1}$. We do not list the well-known relations (see [FGM, Section 1.5] for full details in the notation we use here). Let $U_{q}^{+}(\mathfrak{g})\left(U_{q}^{-}(\mathfrak{g})\right)$ 
be the subalgebra of $U_{q}(\mathfrak{g})$ generated by $E_{i}\left(F_{i}\right), i \in I$, and let $U_{q}^{0}(\mathfrak{g})$ denote the subalgebra generated by $K_{i}^{ \pm}(i \in I)$ and $D^{ \pm}$.

Beck and Kac [BK] have constructed a PBW basis on $\left\{E_{\beta} \mid \beta \in \Delta\right\}$, where we count with multiplicity for the root vectors corresponding to imaginary roots, labeling them $E_{k \delta}^{(i)}$, when we need to distinguish particular root vectors. This basis has several useful properties. The total ordering of the root system induces a total ordering on the basis and the basis is convex, meaning that if $\alpha, \beta$ are positive roots and $E_{\beta}>E_{\alpha}$, that is, $\beta>\alpha$, then

$$
E_{\beta} E_{\alpha}-q^{(\alpha \mid \beta)} E_{\alpha} E_{\beta}=\sum_{\alpha<\gamma_{1}<\cdots<\gamma_{r}<\beta} c_{\gamma} E_{\gamma_{1}}^{a_{1}} \cdots E_{\gamma_{r}}^{a_{r}}
$$

for some integers $a_{1}, \ldots, a_{r}$ and scalars $c_{\gamma} \in \mathbb{C}\left[q, q^{-1}\right], \gamma=\left(\gamma_{1}, \ldots, \gamma_{r}\right)$ [BK, Proposition 1.7c], and similarly for the negative roots. Furthermore, Beck and Kac introduced a notion of total degree of basis elements which provides a filtration of $U_{q}(\mathfrak{g})$ such that the commutation relations for the associated graded algebra are particularly simple. We state their result here as we need to use the commutation relations later.

PROPOSITION 2.1 ([BK, Proposition 1.8]). The associated graded algebra $\operatorname{Gr}_{g}(\mathfrak{g})$ of $U_{q}(\mathfrak{g})$ is the algebra over $\mathbb{C}(q)$ generated by $E_{\alpha}, \alpha \in \Delta$, counting multiplicities, $K_{i}^{ \pm 1}(i \in I)$ and $D^{ \pm}$subject to the relations

$$
\begin{aligned}
& K_{i} K_{i}^{-1}=K_{i}^{-1} K_{i}=D D^{-1}=D^{-1} D=1, \\
& K_{i} K_{j}=K_{j} K_{i}, \quad K_{i} D=D K_{i}, \\
& K_{i} E_{\alpha}=q^{\left(\alpha_{i} \mid \alpha\right)} E_{\alpha} K_{i}, \quad D E_{\alpha}=q^{n} E_{\alpha} D, \quad \text { for } \alpha=\gamma+n \delta, \gamma \in \dot{\Delta} . \\
& E_{\alpha} E_{-\beta}=E_{-\beta} E_{\alpha} \quad \text { if } \alpha, \beta \in \Delta_{+}, \\
& E_{\alpha} E_{\beta}=q^{(\alpha \mid \beta)} E_{\beta} E_{\alpha}, \quad E_{-\alpha} E_{-\beta}=q^{(\alpha \mid \beta)} E_{-\beta} E_{-\alpha}, \quad \text { if } \alpha, \beta \in \Delta_{+} \text {and } \beta<\alpha .
\end{aligned}
$$

Let $S$ be a nonempty subset of $\Delta$. Set $-S=\{-\alpha \mid \alpha \in S\}$. A partition $\Delta=S U-S$ of the root system is called closed if whenever $\alpha$ and $\beta$ are in $S$ and $\alpha+\beta$ is a root, then $\alpha+\beta \in S$. Affine Kac-Moody algebras have a finite number (more than one) of inequivalent Weyl-orbits of closed partitions of the root system. These non-standard closed partitions were first studied and classified by Jakobsen and Kac [JK1, JK2] and independently in [Fu1, Fu2].

Let $J \subseteq \dot{I}=\{1, \ldots, N\}$. Let $\Pi^{J}=\left\{\alpha_{j} \in \Pi \mid j \in J\right\}$. Set $Q^{J}=\oplus_{j \in J} \mathbb{Z} \alpha_{j} \oplus \mathbb{Z} \delta$, and $Q_{ \pm}^{J}=Q^{J} \cap Q_{ \pm}$. We define a partial order on $Q$ by setting $\mu \leq J \lambda$ if $\lambda-\mu \in Q_{+}^{J}$. Let $\dot{\Delta}^{J}$ be the finite root system generated by the simple roots in $\Pi^{J}$. Set

$$
\Delta^{J}=\left\{\alpha+n \delta \in \Delta \mid \alpha \in \dot{\Delta}^{J}, n \in \mathbb{Z}\right\} \cup\{k \delta \mid k \in \mathbb{Z} \backslash\{0\}\},
$$


and let $\Delta_{ \pm}^{J}=\Delta^{J} \cap \Delta_{ \pm}$and $\Delta_{J}^{\infty}=\Delta \backslash \Delta^{J}$. Now we let

$$
{ }_{+} \Delta_{J}^{\infty}=\left\{\alpha+n \delta \in \Delta \mid \alpha \in \dot{\Delta}_{+} \backslash \dot{\Delta}^{J}, n \in \mathbb{Z}\right\} .
$$

Finally, let $S_{J}=\Delta_{+}^{J} U_{+} \Delta_{J}^{\infty}$. Then the sets $S_{J}$ parametrize the closed partitions of $\Delta$ [Fu4, Theorem 2.4]. Note that, if $J=\dot{I}$, then $S_{J}=\Delta_{+}$, the usual partition.

Next we construct quantum Verma-type modules. Let $J \subset i$ and set $S=S_{J}$. Let $\mathfrak{n}^{ \pm J}$ be the subalgebra of $U_{q}(\mathfrak{g})$ generated by $\left\{E_{\beta} \mid \beta \in \pm S\right\}$, and let $\mathfrak{b}^{J}$ denote the subalgebra of $U_{q}(\mathfrak{g})$ generated by $\left\{E_{\beta} \mid \beta \in S\right\} \cup U_{q}^{0}$.

A $U_{q}(\mathfrak{g})$-module $V$ is called a quantum weight module if $V=\oplus_{\mu \in P} V_{\mu}$, where

$$
V_{\mu}=\left\{v \in V \mid K_{i}^{ \pm 1} v=q_{i}^{ \pm \mu\left(h_{i}\right)} v, D^{ \pm 1} v=q_{0}^{ \pm \mu(d)} v\right\}
$$

and we set $q_{i}=q^{\left(\alpha_{i}, \alpha_{i}\right) / 2}$, for $i=0, \ldots, N$. Any submodule of a quantum weight module is a weight module. A $U_{q}(\mathfrak{g})$-module $V$ is called a $J$-highest weight module with highest weight $\lambda \in P$ if there is a non-zero vector $v \in V$ such that

(i) $u v=0$ for all $u \in \mathfrak{n}^{j} \backslash \mathbb{C}(q)^{*}$;

(ii) for each $i \in I, K_{i}^{ \pm 1} v=q_{i}^{ \pm \lambda\left(h_{i}\right)} v, D^{ \pm 1} v=q_{0}^{ \pm \lambda(d)} v$;

(iii) $V=U_{q}(\mathfrak{g}) v$.

In the absence of a general quantum PBW theorem for non-standard partitions, we cannot immediately claim that a $J$-highest weight module $V$ is generated by $\mathfrak{n}^{-J}$.

Let $\mathbb{C}(q) v$ be a 1-dimensional vector space. Let $\lambda \in P$, set $E_{\beta} v=0$ for all $\beta \in S$, $K_{i}^{ \pm 1} v=q_{i}^{ \pm \lambda\left(h_{i}\right)} v(i \in I)$ and $D^{ \pm 1} v=q_{0}^{ \pm \lambda(d)} v$. Now define $M^{J}(\lambda)=U_{q}(\mathfrak{g}) \otimes_{\mathfrak{b}}, \mathbb{C}(q) v$. Then $M^{J}(\lambda)$ is a $J$-highest weight $U_{q}(\mathfrak{g})$-module called the quantum Verma-type module with highest weight $\lambda$. Quantum Verma-type modules have many similar properties to ordinary quantum Verma modules except that they typically have both finite- and infinite-dimensional weight spaces.

Basic properties of quantum Verma-type modules were studied in [FGM], where it was also shown that the quantum Verma-type modules are true quantum deformations of Verma-type modules for the underlying affine algebra. In order to prove the equivalence of categories, we need a number of results from [FGM], which we collect here for the convenience of the reader. The most important of these results are the following, which give an explicit description of a basis for the quantum Verma-type modules, and a determination of their finite and infinite-dimensional weight spaces.

PROPOSITION 2.2 ([FGM, Theorem 3.5]). As a vector space over $\mathbb{C}(q), M^{J}(\lambda)$ is isomorphic to the space spanned by the ordered monomials

$$
E_{-\beta-n \delta} \cdots E_{-\beta+k \delta} \cdots E_{-\alpha-n \delta} \cdots E_{-k \delta} \cdots E_{\alpha-k \delta},
$$

for $\alpha \in \dot{\Delta}_{+} \cap \Delta^{J}, \beta \in \dot{\Delta}_{+} \cap \Delta_{J}^{\infty}, n \geq 0, k>0$. 
Proposition 2.3 ([FGM, Proposition 5.5]). Let $\lambda \in P, J \subseteq i$, and let $M^{J}(\lambda)$ be the quantum Verma-type module with highest weight $\lambda$. Then $\operatorname{dim} M^{J}(\lambda)_{\mu} \neq 0$ if and only if $\lambda-\mu$ is in the monoid generated by $S_{J}, \operatorname{dim} M^{J}(\lambda)_{\lambda}=1$, and $0<$ $\operatorname{dim} M^{J}(\lambda)_{\mu}<\infty$ if and only if $\mu \leq_{J} \lambda$.

Consider the following subsets of $\Delta$ :

$$
\begin{array}{ll}
A_{1}=\left\{\alpha+n \delta \mid \alpha \in \dot{\Delta}_{+}, n \geq 0\right\}, & B_{1}=\left\{-\alpha-n \delta \mid \alpha \in \dot{\Delta}_{+}, n \geq 0\right\}, \\
A_{2}=\{k \delta \mid k>0\}, & B_{2}=\{-k \delta \mid k>0\}, \\
A_{3}=\left\{-\alpha+k \delta \mid \alpha \in \dot{\Delta}_{+}, k>0\right\}, & B_{3}=\left\{\alpha-k \delta \mid \alpha \in \dot{\Delta}_{+}, k>0\right\} .
\end{array}
$$

Then $\Delta_{+}=A_{1} \cup A_{2} \cup A_{3}$ and $\Delta_{-}=B_{1} \cup B_{2} \cup B_{3}$. Further, in the total ordering of the root system, we have

$$
B_{1}<B_{2}<B_{3}<A_{3}<A_{2}<A_{1} .
$$

Next, we split these subsets into those associated with finite and infinite-dimensional subspaces. For $i=1,2,3$, let $A_{i}^{f i n}=A_{i} \cap \Delta^{J}, A_{i}^{\infty}=A_{i} \cap \Delta_{J}^{\infty}, B_{i}^{f i n}=B_{i} \cap \Delta^{J}$ and $B_{i}^{\infty}=B_{i} \cap \Delta_{J}^{\infty}$. Now, let $X_{i}$ denote an ordered monomial in elements $E_{\beta}, \beta \in A_{i}$, and $Y_{i}$ denote an ordered monomial of elements $E_{\beta}, \beta \in B_{i}$. Further, let $X_{i}^{f i n}\left(X_{i}^{\infty}\right)$ denote an ordered monomial in $E_{\beta}, \beta \in A_{i}^{f i n}\left(\beta \in A_{i}^{\infty}\right)$, and let $Y_{i}^{f i n}\left(Y_{i}^{\infty}\right)$ denote an ordered monomial in $E_{\beta}, \beta \in B_{i}^{f i n}\left(\beta \in B_{i}^{\infty}\right)$. We note that the sets $A_{2}^{\infty}$ and $B_{2}^{\infty}$ are actually empty.

We now define a number of subalgebras of the quantum group. Let $\mathfrak{p}^{J}$ be the subalgebra of $U_{q}(\mathfrak{g})$ generated by monomials of the form $X_{1}^{\infty}, Y_{3}^{\infty}, X_{i}^{f i n}, Y_{i}^{f i n}$ for $i=1,2,3$ and $U_{q}^{0}(\mathfrak{g})$. Let $\mathfrak{g}^{J}$, be the subalgebra generated by monomials of the form $X_{i}^{f i n}$ and $Y_{i}^{f i n}(i=1,2,3)$ and $U_{q}^{0}(\mathfrak{g})$ and let $\tilde{\mathfrak{g}}^{f}$ denote the algebra generated by monomials of the form $X_{1}^{f i n}, X_{3}^{f i n}, Y_{1}^{f i n}$ and $Y_{3}^{f i n}$ together with $U_{q}^{0}(\mathfrak{g})$. We note that $\mathfrak{g}^{J}$ and $\tilde{\mathfrak{g}}^{f}$ are subalgebras of $\mathfrak{p}^{J}$. As we will next be considering $\mathfrak{g}^{J}$-modules, we now define $\mathfrak{b}_{J}=\mathfrak{g}^{J} \cap \mathfrak{b}^{J}$.

\section{Representations of $\mathfrak{g}^{J}$}

Let $U^{J}$ be the $\mathbb{C}(q)$-linear subspace of $U_{q}(g)$ spanned by ordered monomials of the form $Y_{3}^{\infty}$ and $X_{1}^{\infty}$, and $U^{-J}$ be the $\mathbb{C}(q)$-linear subspace spanned by monomials of the form $Y_{1}^{\infty}$ and $X_{3}^{\infty}$.

Consider the subspace of the Verma-type module $M^{J}(\lambda)$ given by $\Omega=\mathbb{C}(q)$ $\operatorname{span}\left\{E_{-\alpha-n \delta} \cdots E_{-k \delta} \cdots E_{\alpha-k \delta} v_{\lambda} \mid \alpha \in \dot{\Delta}_{+} \cap \Delta^{J}, n \geq 0, k>0\right\}$.

PROPOSITION 3.1. The subspace $\Omega$ is a $\mathfrak{g}^{J}$-submodule of $M^{J}(\lambda)$. 
PROOF. Note that all the root vectors in the monomials spanning $\Omega$ are from finitedimensional root spaces. Similarly, the generators of the subalgebra $\mathfrak{g}^{J}$ are also monomials in root vectors from finite-dimensional root spaces. Let one of these generators act on a vector in $\Omega$. By Proposition 2.2, we may reorder the resulting vector. But by the root grading, there cannot be any monomials from the infinitedimensional root spaces in the final result. Hence, $\Omega$ is stabilized by $\mathfrak{g}^{j}$.

Denote by $M^{f}(\lambda)$ the space $\Omega$ when viewed as a $\mathfrak{g}^{J}$-module. Then $M^{f}(\lambda)$ can be considered as a Verma module for $\mathfrak{g}^{J}$ with respect to $\mathfrak{b}_{J}$ with highest weight $\lambda$. For any submodule $N$ of $M^{J}(\lambda)$, let $N^{f}:=N \cap M^{f}(\lambda)$. Then $N^{f}$ is a $\mathfrak{g}^{J}$-module and also a $\mathfrak{p}^{J}$-module with trivial action of monomials of the form $X_{1}^{\infty}$ and $Y_{3}^{\infty}$ (this follows from the root grading of $M^{J}(\lambda)$ [FGM, Theorem 3.5]). It follows that $M^{J}(\lambda) \cong U_{q}(\mathfrak{g}) \otimes_{\mathfrak{p}^{\prime}} M^{f}(\lambda)$ from the universality of the tensor product.

PROPOSITION 3.2 ([FGM, Theorem 6.4]). Let $N$ be a non-trivial submodule of $M^{J}(\lambda)$. Then

(i) $N^{f} \neq 0$, and

(ii) if $\lambda(c) \neq 0$, then as vector spaces, $N \cong U^{-J} \otimes N^{f}$.

We also note that, with the notation above, $M^{J}(\lambda) / N \cong U^{-J} \otimes\left(M^{f}(\lambda) / N^{f}\right)$ as vector spaces.

\section{Representations of quantum Heisenberg algebras}

Let $G_{q}$ be the subalgebra of $U_{q}(\mathfrak{g})$ generated by monomials of the form $X_{2}$ and $Y_{2}$. Since $\left[E_{k \delta}^{(i)}, E_{l \delta}^{(j)}\right]=0$ if $k \neq-l$ and $\left[E_{k \delta}^{(i)}, E_{-k \delta}^{(j)}\right] \in Z$ for some space $Z$ generated by certain elements $z^{ \pm}$, central in $U_{q}(\mathfrak{g})$ (see [Be, Lemma 4.5], or [KT, Proposition 3.2] for precise description of the elements $z^{ \pm}$), the algebra $G_{q}$ is a quantum Heisenberg algebra [FO]. We set $G_{q}^{ \pm}=G_{q} \cap U_{q}^{ \pm}(\mathfrak{g})$. The Heisenberg algebra $G_{q}$ has a natural $\mathbb{Z}$-grading with $\operatorname{deg} z^{ \pm}=0$ and $\operatorname{deg} E_{k \delta}^{(j)}=k$ for all $k \in \mathbb{Z} \backslash\{0\}, j \in I$. A $G_{q^{-}}$ module $V$ is called $\mathbb{Z}$-graded if $V=\oplus_{i \in \mathbb{Z}} V_{i}$, where $z^{ \pm} V_{i} \subseteq V_{i}$ and $E_{k \delta}^{(j)} V_{i} \subseteq V_{k+i}$ for all $k \in \mathbb{Z} \backslash\{0\}, j \in I$ and $i \in \mathbb{Z}$. Let $v_{\lambda}$ be a generating vector for the quantum Verma-type module $M^{J}(\lambda)$ with $J$-highest weight $\lambda \in P$. Then the $G_{q}$-module $M_{G}(\lambda):=G_{q} v_{\lambda}$ is a Verma-module for $G_{q}$.

We now recall from [FGM] the construction of a certain subalgebra $\bar{G}_{q}$ of $G_{q}$. In the affine algebra $\mathfrak{g}$ underlying $U_{\mathfrak{q}}(\mathfrak{g})$ is a corresponding Heisenberg algebra $G=$ $\oplus_{k \in \mathbb{Z} \backslash(0\}} \mathfrak{g}_{k \delta} \oplus \mathbb{C} \mathcal{c}$, with subalgebras $G^{ \pm}=\oplus_{k>0} \mathfrak{g}_{ \pm k \delta}$. We call a subset $C \subseteq J$ connected if the Coxeter-Dynkin diagram associated to the simple roots $\alpha_{i}, i \in C$, is connected. The set $J$ can then be partitioned into a collection $\mathscr{C}$ of subsets corresponding to connected components of the Coxeter-Dynkin diagram associated to $J$. For $C \in \mathscr{C}$, 
let $\mathfrak{A}_{C}$ be the affine subalgebra of $\mathfrak{g}$ generated by $e_{i}, f_{i}(i \in C)$, together with the central element $c$ and degree derivation $d$ from $\mathfrak{g}$. Let $\mathfrak{g}_{C}=\sum_{C \in \mathscr{C}} \mathfrak{A}_{C}$. Set $\bar{G}=\mathbb{C}-\operatorname{span}\left\{g \in G^{-} \oplus G^{+} \mid\left[g, \mathfrak{g}_{C}\right]=0\right\}$, and set $\bar{G}^{ \pm}=\bar{G} \cap G^{ \pm}$. Then $\bar{G}^{ \pm}$are subalgebras of $G$.

An element $x \in \bar{G}$ consists of $\mathbb{C}$-linear combinations of imaginary root vectors $e_{k \delta}^{(i)}$, where the $e_{\beta}$ are a (particular) PBW basis of $U(\mathfrak{g})$. Write $x=\sum_{1 \leq i \leq N, k \in \mathbb{Z}} c_{i, k} e_{k \delta}^{(i)}$. For each $k \in \mathbb{Z}$ and $i=1, \ldots, N$, by Beck's construction of the PBW basis [Be] there is a root vector $E_{k \delta}^{(i)}$ in $U_{q}(g)$ such that the classical limit of $E_{k \delta}^{(i)}$ may be identified with $e_{k \delta}^{(i)}$. Consider the element $X=\sum_{1 \leq i \leq N, k \in \mathbb{Z}} c_{i, k} E_{k \delta}^{(i)}$ in $U_{q}(\mathfrak{g})$. Since the coefficients of the $E_{k \delta}^{(i)}$ 's are in $\mathbb{C}$, certainly $X \in U_{\mathrm{A}}$, the $\mathbb{A}$-form of $U_{q}(\mathfrak{g})$ (see [FGM, Section 4] for details on the ring $\mathbb{A}=\mathbb{C}\left[q, q^{-1}, 1 /[n]_{q_{i}}, i \in I, n>0\right]$, the $\mathbb{A}$-form construction and the quantum deformation process) and the classical limit of $X$ may be identified with $x \in \bar{G}$. Write $X=\phi(x)$.

Define $\bar{G}_{q}$ to be the unital subalgebra of $G_{q}$

$$
\left.\bar{G}_{q}=\langle f(q) X| f(q) \in \mathbb{C}(q), X=\phi(x) \text { for some } x \in \bar{G}\right\rangle .
$$

Similarly, we set

$$
\left.\bar{G}_{q}^{ \pm}=\langle f(q) X| f(q) \in \mathbb{C}(q), X=\phi(x) \text { for some } x \in \bar{G}^{ \pm}\right\rangle .
$$

We note that $\left[\bar{G}_{q}, \tilde{\mathfrak{g}}^{f}\right]=0$ and $\mathfrak{g}^{J} \cong \bar{G}_{q}^{-} \otimes \tilde{\mathfrak{g}}^{f} \otimes \bar{G}_{q}^{+}$, ([FGM, Proposition 6.1]). Further, the following result is well-known (see, for example [FGM, Proposition 6.2]).

PROPOSITION 4.1. Let $\lambda(c) \neq 0$. The $G_{q}$-Verma module $M_{G}(\lambda)$ is irreducible.

COROLLARY 4.2. The above result also holds with $G_{q}$ replaced by $\bar{G}_{q}$.

\section{The category $\mathscr{O}^{J}(\lambda)$}

We now fix $\lambda \in P$ such that $\lambda(c) \neq 0$. Throughout the remainder of this paper we assume $J \neq \emptyset$ and $J \neq \dot{I}$. If $J=\dot{I}$, then Verma-type modules are just the ordinary Verma modules. But in this case every weight-space is finite-dimensional and there is nothing to prove. At the other extreme, when $J=\emptyset$, the Verma-type modules are imaginary Verma modules. The category we define for the Heisenberg algebra is then the category of ordinary Verma modules, and these are all irreducible for $\lambda(c) \neq 0$. Hence, the Verma modules are the only objects in the category and the analysis used below for the general case is not necessary. The structure of imaginary Verma modules was considered in [FGM].

As $\lambda \in P$ and $J$ are fixed, we shall suppress them in the notation below whenever no confusion will arise. 
Let $\mathscr{O}^{f}(\lambda)$ denote the full subcategory of the category of weight $g^{J}$-modules such that the objects $V$ have all finite-dimensional weight spaces and, if $V_{\mu}$ is a weight space of $V$, then $\mu \leq_{J} \lambda$. Next, we define a category $\mathscr{O}^{J}(\lambda)$ to be the category of those $\mathfrak{p}^{J}$-modules $V$ such that $V \in \mathscr{O}^{f}(\lambda)$ as a $\mathfrak{g}^{J}$-module and such that $X_{1}^{\infty}$ and $Y_{3}^{\infty}$ act trivially. We note that the categories $\mathscr{O}^{f}(\lambda)$ and $\mathscr{O}^{J}(\lambda)$ may be equivalent, but we do not have a proof.

As in the classical case, the categories $\mathscr{O}^{J}(\lambda)$ and $\mathscr{O}^{f}(\lambda)$ are closed under the operations of taking submodules, quotients and finite direct sums. Also, the (ordinary) quantum Verma modules $M^{f}(\mu)$ of $\mathfrak{g}^{J}$ and their irreducible quotients are in $\mathscr{O}^{f}(\lambda)$ for any $\mu$ such that $\mu \leq_{J} \lambda$.

Let $A$ be an algebra, $B$ a subalgebra of $A$ and $M$ an $A$-module. We call a $B$-module $V$ a highest weight module with respect to $B$ if there is some $\lambda \in B^{*}$ and vector $v \in V$ such that $V$ is generated by $v$ and $b v=\lambda(b) v$ for all $b \in B$. A highest weight series for $M$ with respect to $B$ is a sequence of submodules

$$
\text { (0) } \subset M_{1} \subset M_{2} \subset M_{3} \subset \cdots
$$

of $M$ such that $M=\bigcup_{i=0}^{\infty} M_{i}$ and $M_{i} / M_{i-1}$ is a highest-weight module with respect to $B$ for each $i$.

LEMMA 5.1. Every $\mathfrak{p}^{J}$-module $V$ in $\mathscr{O}^{J}(\lambda)$ has a highest weight series with respect to $\mathfrak{b}_{J}$.

PROOF. A similar argument to that of Lemma 5.1 of [RW] shows that all $\mathfrak{g}^{\prime}$-modules in $\mathscr{O}^{f}(\lambda)$ have highest weight series. Hence the same is true for all modules $V$ in $\mathscr{O}^{J}(\lambda)$ when viewed as $\mathfrak{g}^{J}$-modules. Since the action of $X_{1}^{\infty}$ and $Y_{3}^{\infty}$ is trivial, as a $\mathfrak{p}^{J}$-module, $V$ has a highest weight series with respect to $\mathfrak{b}_{J}$.

For any non-trivial $\mathfrak{g}^{J}$-module $M \in \mathscr{O}^{f}(\lambda)$ we define the subspace $M^{\bar{G}_{q}^{+}}=\{v \in$ $M \mid f v=0$ for all $\left.f \in \bar{G}_{q}^{+} \backslash \mathbb{C}(q)\right\}$. Then $M^{\bar{G}_{q}^{+}}$is a non-trivial $\tilde{\mathfrak{g}}^{f}$-module as $\left[\tilde{\mathfrak{g}}^{f}, \bar{G}_{q}^{+}\right]=0$.

PROPOSITION 5.2. Let $\lambda(c) \neq 0$. If $V \in \mathscr{O}^{J}$, then $V$ is completely reducible as a $G_{q^{-}}$ module with irreducible components isomorphic to $M_{G}(\lambda)$. Moreover, $V \cong \bar{G}_{q}^{-} \otimes V^{\bar{G}_{q}^{+}}$ as vector spaces.

Proof. Let $V \in \mathscr{O}^{J}$. Consider $V^{+}:=V^{\bar{G}_{q}^{+}}$. It follows from [FO, Theorem 2.4] that $V$ is completely reducible as a $G_{q}$-module with irreducible components isomorphic to $M_{G}(\lambda)$, up to a shift in gradation. This also implies $V=\bar{G}_{q} V^{+}$. Using Proposition 4.1 we conclude that $V \cong \bar{G}_{q}^{-} \otimes V^{+}$. 


\section{An equivalence of categories}

In this section, we define an equivalence of certain categories of weight $U_{q}(\mathfrak{g})$ modules. First, we define $\mathscr{O}_{J}(\lambda)$ to be the full subcategory of the category of weight $U_{q}(\mathfrak{g})$-modules with objects those weight modules $V$ such that, if $V_{\mu}$ is a weight space of $V$, then $\lambda-\mu$ is in the monoid generated by $S_{J}$, and $V$ is generated by the $g^{J}$-module $V^{f}=\sum V_{\mu}, \mu \leq_{J} \lambda$. By [FGM, Proposition 5.5], for any $\lambda \in P$, the Verma-type modules $M^{J}(\mu)$ are in $\mathscr{O}_{J}(\lambda)$ for all $\mu \leq_{J} \lambda$. We will show that the categories $\mathscr{O}_{J}(\lambda)$ and $\mathscr{O}^{J}(\lambda)$ are equivalent.

If $V \in \mathscr{O}_{J}(\lambda)$, then the $\mathfrak{g}^{J}$-module $V^{f} \in \mathscr{O}^{J}(\lambda)$. Define a functor $F: \mathscr{O}_{J}(\lambda) \rightarrow$ $\mathscr{O}^{J}(\lambda)$ by $F(V)=V^{f}$ and $F(f)=\left.f\right|_{V^{\prime}}$ for any $V \in \mathscr{O}_{J}(\lambda)$ and any $f \in \operatorname{Hom}\left(V, V^{\prime}\right)$ in $\mathscr{O}_{J}(\lambda)$. Note that monomials of the form $X_{1}^{\infty}$ and $Y_{3}^{\infty}$ act trivially on $V^{f}$ since all weight spaces $V_{\mu}^{f}$ of $V^{f}$ have $\mu \leq \lambda \lambda$. Since $V$ is generated by $V^{f}$, the functor $F$ is exact.

Let $N \in \mathscr{O}^{J}(\lambda)$. Then $N$ is a $\mathfrak{p}^{J}$-module, such that monomials of the form $X_{1}^{\infty}$ and $Y_{3}^{\infty}$ act trivially. We define a functor $Y: \mathscr{O}^{J}(\lambda) \rightarrow \mathscr{O}_{J}(\lambda)$ by $Y(N)=U_{q}(g) \otimes_{p^{J}} N$ and $Y(f)=1 \otimes f$ for any $f \in \operatorname{Hom}\left(N, N^{\prime}\right)$ and any objects $N, N^{\prime} \in \mathscr{O}^{J}(\lambda)$.

PROPOSITION 6.1. Let $V \in \mathscr{O}^{J}(\lambda)$. Then $Y(V) \cong U^{-J} \otimes_{\mathrm{C}(q)} V$ as vector spaces. In particular, $Y$ is an exact functor.

PROOF. The argument here is similar to that used in proving Theorem 3.5 in [FGM] and we summarize some technical details here where they are substantially identical to those used in that proof.

Let $V$ be a $\mathfrak{p}^{J}$-module such that $U^{J}$ acts trivially. Then $V$ is a $\mathfrak{g}^{J}$-module and $Y(V)=U_{q}(\mathfrak{g}) \otimes_{\mathfrak{p}}, V$, where monomials of the form $X_{1}^{\infty}$ and $Y_{3}^{\infty}$ in $\mathfrak{p}^{J}$ act trivially on $V$.

Using Beck's PBW basis, any element $u \in U_{q}(\mathfrak{g})$ may be written in the form

$$
u=\sum Y_{1} Y_{2} Y_{3} Z X_{3} X_{2} X_{1},
$$

where $Z \in U_{q}^{0}(\mathfrak{g})$. Consider a typical subspace $W$ of $Y(V)$ of the form

$$
W=Y_{1} Y_{2} Y_{3} Z X_{3} X_{2} X_{1} \otimes_{p}, V
$$

First, we note that all monomials $X_{1}$ and $X_{2}$ are in $\mathfrak{p}^{J}$ and $Z$ commutes with any monomial $X_{3}$ up to a scalar in $\mathbb{C}(q)$. Hence, we may write

$$
W=Y_{1} Y_{2} Y_{3} X_{3} \otimes_{p^{\prime}} V
$$

To proceed, we rearrange the monomials in steps, arguing by induction on the Beck$\mathrm{Kac}$ total degree. The base of the induction is trivial as in that case there is only one 
simple root involved and nothing to do. We suppose the theorem is true for monomials up to total degree $d$ and consider monomials of next highest degree.

As monomials of the form $X_{3}^{f i n}$ are all in $\mathfrak{p}^{j}$, we need to show we can move any such terms to the right of any terms from $X_{3}^{\infty}$. As argued in [FGM, Theorem 3.5], if we attempt to replace factors of the form $E_{-\alpha+k \delta} E_{-\beta+l \delta}$ (with $-\alpha+k \delta \in A_{3}^{f i n}$ and $-\beta+l \delta \in A_{3}^{\infty}$ ) in a monomial $X_{3}$ with the better ordered factors $E_{-\beta+l \delta} E_{-\alpha+k \delta}$, a consideration of the convexity properties of the basis shows that any additional monomials generated will either have lower total degree (and so be rearrangeable by inductive hypothesis) or have the same total degree, but fewer terms in $X_{3}^{f \text { in }}$. Repeating the process as necessary, we are reduced to considering spaces of the form $Y_{1} Y_{2} Y_{3} X_{3}^{\infty} \otimes_{p}, V$.

The arguments for commuting monomials of the form $Y_{3}$ and $Y_{2}$ with $X_{3}^{\infty}$, writing monomials $Y_{1}$ in the form $Y_{1}^{\infty} Y_{1}^{f i n}$ and commuting $Y_{1}^{f \text { in }}$ with $X_{3}^{\infty}$, now proceed exactly as in the proof of [FGM, Theorem 3.5]. The monomials $Y_{3}, Y_{2}$ and $Y_{1}^{f \text { in }}$ are all in $\mathfrak{p}^{j}$, and so we have shown that $U_{q}(\mathfrak{g}) \otimes_{\mathfrak{p}}, V$ can be viewed as a subspace of $Y_{1}^{\infty} X_{3}^{\infty} \otimes_{\mathfrak{p}}, V$.

Now, suppose some monomial of the form $X_{3}^{\infty}$ appears in $\mathfrak{p}^{J}$. Then, due to the root grading on $\mathfrak{p}^{J}$, there must also be a counterbalancing monomial of the form $Y_{3}^{\infty}$. Commuting if necessary, such terms must disappear as $Y_{3}^{\infty} \in \mathfrak{p}^{J}$ and acts as 0 on $V$. A similar argument applies to monomials of the form $Y_{1}^{\infty}$. Hence, by the universality of $U_{q}(\mathrm{~g})$, we have $Y(V) \cong U^{-J} \otimes_{\mathrm{C}(q)} V$.

COROLlaRY 6.2. Let $N$ be a submodule of a Verma-type module. Then $N \cong$ $U_{q}(\mathfrak{g}) \otimes_{\mathfrak{p}} N^{f}$ as $U_{q}(\mathfrak{g})$-module.

Proof. Let $N$ be a submodule of a Verma-type module $M^{J}(\lambda)$. It follows from Proposition 3.2 that $N \cong U^{-J} \otimes N^{f}$. Now $N^{f} \in \mathscr{O}^{J}(\lambda)$ and so, by Proposition 6.1, $Y\left(N^{f}\right) \cong U^{-J} \otimes N^{f}$ as vector spaces. Hence, $N \cong Y\left(N^{f}\right)$ as vector spaces. But $N$ is a homomorphic image of $Y\left(N^{f}\right)$ by the universality of the tensor product. Hence, $N \cong Y\left(N^{f}\right)=U_{q}(\mathfrak{g}) \otimes_{\mathfrak{p}^{\prime}} N^{f}$ as $U_{q}(\mathfrak{g})$-modules.

Proposttion 6.3. Let $V \in O_{J}$. Then $V$ has a highest weight series $\left\{M_{i}\right\}$ such that for each $i, M_{i} / M_{i-1} \cong M^{J}\left(\mu_{i}\right) / Y\left(N_{i}\right)$ for some $\mu_{i} \in P$ and $N_{i} \in O^{J}$.

Proof. Let $V \in \mathscr{O}_{J}(\lambda)$. Then $F(V)=V^{f} \in \mathscr{O}^{J}(\lambda)$ and, by Lemma 5.1, $V^{f}$ has a highest weight series $\left\{K_{i}\right\}$ with respect to $\mathfrak{b}_{J}$. Set $M_{i}=U_{q}(\mathfrak{g}) K_{i}$ for each $i$. Each $K_{i}$ is a submodule of $V^{f}$ and $V$ is generated by $V^{f}$ since $V \in \mathscr{O}_{J}(\lambda)$, so each $M_{i}$ is a submodule of $V$.

Consider $Y\left(K_{i}\right)=U_{q}(\mathfrak{g}) \otimes_{\mathfrak{p}}, K_{i}$. By the universality of $U_{q}(\mathfrak{g})$, there is a surjective homomorphism $\phi_{i}: Y\left(K_{i}\right) \rightarrow M_{i}$.

Since $\left\{K_{i}\right\}$ is a highest weight series for $V^{f}$, each $K_{i} / K_{i-1}$ is a $J$-highest weight module with respect to $b_{J}$ with some highest weight $\mu_{i}$. Then $Y\left(K_{i} / K_{i-1}\right)$ is a highest- 
weight $U_{q}(\mathfrak{g})$-module with highest weight $\mu_{i}$ with respect to $\mathfrak{b}^{J}$, and by the universality of Verma-type modules, there is a surjective homomorphism $M^{J}\left(\mu_{i}\right) \rightarrow Y\left(K_{i} / K_{i-1}\right)$. By Proposition 6.1, the functor $Y$ is exact, so $Y\left(K_{i} / K_{i-1}\right) \cong Y\left(K_{i}\right) / Y\left(K_{i-1}\right)$, and hence we have a surjective homomorphism $\psi_{i}: M^{J}\left(\mu_{i}\right) \rightarrow Y\left(K_{i}\right) / Y\left(K_{i-1}\right)$.

The homomorphism $\psi_{i}$ induces a surjective homomorphism $\Psi_{i}: M^{J}\left(\mu_{i}\right) \rightarrow$ $M_{i} / M_{i-1}$. Consider $\operatorname{ker} \Psi_{i}$. If $\operatorname{ker} \Psi_{i}=(0)$ then it is trivially in $\mathscr{O}_{J}(\lambda)$. If $\operatorname{ker} \Psi_{i}$ is a non-trivial submodule of $M^{J}\left(\mu_{i}\right)$, then by [FGM, Theorem 6.4] it is generated by $\left(\operatorname{ker} \Psi_{i}\right)^{f}$ and so in $\mathscr{O}_{J}(\lambda)$ (the other conditions of membership are clearly satisfied). By Corollary $6.2, Y \circ F\left(\operatorname{ker} \Psi_{i}\right) \cong \operatorname{ker} \Psi_{i}$. Set $N_{i}=F\left(\operatorname{ker} \Psi_{i}\right)$. Then we have $Y\left(N_{i}\right) \cong \operatorname{ker} \Psi_{i}$ and so

$$
M^{J}\left(\mu_{i}\right) / \operatorname{ker} \Psi_{i} \cong M^{J}\left(\mu_{i}\right) / Y\left(N_{i}\right) \cong M_{i} / M_{i-1} .
$$

Now each $M_{i}$ is a submodule of $V$ and $(0) \subset M_{1} \subset M_{2} \subset \cdots$. Further,

$$
\bigcup_{i=0}^{\infty} M_{i}=\bigcup_{i=0}^{\infty} U_{q}(\mathfrak{g}) K_{i}=U_{q}(\mathfrak{g}) \bigcup_{i=0}^{\infty} K_{i}=U_{q}(\mathfrak{g}) V^{f} \cong V,
$$

since $\left\{K_{i}\right\}$ is a highest weight series for $V^{f}$ and $V^{f}$ generates $V$. It is clear that each $M_{i} / M_{i-1}$ is a highest weight module with respect to $\mathfrak{b}^{J}$, and so $\left\{M_{i}\right\}$ is a highest weight series for $V$.

PROPOSITION 6.4. The category $\mathscr{O}_{J}(\lambda)$ is closed under the operations of taking submodules, quotients and finite direct sums.

PROOF. That $\mathscr{O}_{J}(\lambda)$ is closed under taking finite direct sums is clear.

We next prove that any submodule of a module in $\mathscr{O}_{J}(\lambda)$ is itself in $\mathscr{O}_{J}(\lambda)$. Let $M \in \mathscr{O}_{J}(\lambda)$ and let $N$ be a submodule of $M$. Then certainly $N$ is a weight $U_{q}(\mathfrak{g})$ module such that if $\mu$ is a weight of $N$, then $\lambda-\mu$ is in the monoid generated by $S_{J}$ and if $\mu \leq_{J} \lambda$, then $\operatorname{dim} N_{\mu}<\infty$. It remains to show that $N$ is generated by $N^{f}=\sum_{\mu \leq \lambda} N_{\mu}$.

By Proposition 6.3, the $U_{q}(\mathfrak{g})$-module $M$ has a highest weight series $\left\{M_{i}\right\}$ such that each $M_{i} / M_{i-1}$ is a homomorphic image of some quantum Verma-type module $M^{J}\left(\mu_{i}\right)$. Denote $N_{i}=N \cap M_{i}$. Then $\bigcup_{i=0}^{\infty} N_{i}=\bigcup_{i=0}^{\infty}\left(N \cap M_{i}\right)=N \cap \bigcup_{i=0}^{\infty} M_{i}=N \cap M=N$.

Set $\bar{N}_{i}=N_{i} / N_{i-1}$. Then

$$
\begin{aligned}
\bar{N}_{i}=\left(N \cap M_{i}\right) /\left(N \cap M_{i-1}\right) & \cong\left(\left(N \cap M_{i}\right)+M_{i-1}\right) / M_{i-1} \\
& \cong\left(M_{i} \cap\left(N+M_{i-1}\right)\right) / M_{i-1} \subseteq M_{i} / M_{i-1} .
\end{aligned}
$$

We need to show that each $\bar{N}_{i}$ is generated by its finite part. If $\bar{N}_{i}=(0)$, there is nothing to prove. Hence, we may assume $\bar{N}_{i} \neq 0$. Recall from Proposition 6.3 
the surjective homomorphism $\Psi_{i}: M^{J}\left(\mu_{i}\right) \rightarrow M_{i} / M_{i-1}$. Let $L_{i}=\operatorname{ker} \Psi_{i}$, and $K_{i}=\Psi_{i}^{-1}\left(\bar{N}_{i}\right) \subseteq M^{J}\left(\mu_{i}\right)$. Since $K_{i}$ and $L_{i}$ are submodules of a Verma-type module, by Proposition 3.2, $K_{i} \cong U^{-J} \otimes K_{i}^{f}$ and $L_{i} \cong U^{-J} \otimes L_{i}^{f}$ and $\Psi_{i}$ restricts to a surjection $\phi_{i}: U^{-J} \otimes K_{i}^{f} \rightarrow \bar{N}_{i}$. Hence, we have

$$
\begin{aligned}
\bar{N}_{i} & \cong\left(U^{-J} \otimes K_{i}^{f}\right) / \operatorname{ker} \phi_{i}=\left(U^{-J} \otimes K_{i}^{f}\right) /\left(\left(U^{-J} \otimes K_{i}^{f}\right) \cap\left(U^{-J} \otimes L_{i}^{f}\right)\right) \\
& \cong\left(U^{-J} \otimes K_{i}^{f}\right) /\left(U^{-J} \otimes\left(K_{i}^{f} \cap L_{i}^{f}\right)\right) \cong\left(U^{-J} \otimes\left(K_{i}^{f} / K_{i}^{f} \cap L_{i}^{f}\right)\right) .
\end{aligned}
$$

Since $U^{-J}$ is spanned by monomials of the form $X_{1}^{\infty}$ and $Y_{3}^{\infty}$, the finite-dimensional weight spaces of $\bar{N}_{i}$ are generated by vectors of the form $1 \otimes x$ for $x \in K_{i}^{f} / K_{i}^{f} \cap L_{i}^{f}$. Thus, $\bar{N}_{i}^{f} \cong K_{i}^{f} / K_{i}^{f} \cap L_{i}^{f}$ and $\bar{N}_{i}$ is generated as $U_{q}(\mathfrak{g})$-module by $\bar{N}_{i}^{f}$. Hence $\mathscr{O}_{J}(\lambda)$ is closed under taking submodules. It then follows that the category is also closed under the operation of taking quotients. We also note that, if $M, N \in \mathscr{O}_{J}(\lambda)$ and $N \subseteq M$, then $(M / N)^{f} \cong M^{f} / N^{f}$ as vector spaces. In fact, if follows from Corollary 6.6 that they are isomorphic as $\mathfrak{g}^{J}$-modules.

THEOREM 6.5. The categories $\mathscr{O}^{J}(\lambda)$ and $\mathscr{O}_{J}(\lambda)$ are equivalent.

Proof. Let $N \in \mathscr{O}^{J}(\lambda)$. Then $Y(N)=U_{q}(\mathfrak{g}) \otimes_{\mathfrak{p}^{J}} N \cong U^{-J} \otimes N$ as vector spaces by Proposition 6.1. As a $U_{q}(\mathfrak{g})$-module, the finite-dimensional weight spaces of $U^{-J} \otimes N$ are generated by vectors of the form $1 \otimes x$, where $x \in N$ since $U^{-J}$ is generated by monomials of the form $Y_{1}^{\infty}$ and $X_{3}^{\infty}$. Hence $F\left(U^{-J} \otimes N\right)=\left(U^{-J} \otimes\right.$ $N)^{f} \cong N$. That is, $F \circ Y(N) \cong N$ for all $N \in \mathscr{O}^{J}(\lambda)$ and so $F \circ Y$ is naturally equivalent to $I_{\mathscr{O}^{J}(\lambda)}$.

Let $M \in \mathscr{O}_{J}(\lambda)$. By Proposition 6.3, $M$ has a highest weight series $\left\{M_{i}\right\}$ such that $M_{i} / M_{i-1} \cong M^{J}\left(\mu_{i}\right) / Y\left(N_{i}\right)$ for some $\mu_{i}$ and $N_{i} \in \mathscr{O}^{J}(\lambda)$. By Proposition 6.4, each quotient $M_{i} / M_{i-1}$ is in $\mathscr{O}_{J}(\lambda)$. Hence, by Proposition 6.4 and Proposition 3.2, $M_{i} / M_{i-1} \cong U^{-J} \otimes\left(M_{i} / M_{i-1}\right)^{f}$. Then, by Proposition 6.1, $Y \circ F\left(M_{i} / M_{i-1}\right) \cong$ $M_{i} / M_{i-1}$ for all $i$. In particular $Y \circ F\left(M_{1}\right) \cong M_{1}$. Then induction on $i$ and the Five Lemma show that $Y \circ F\left(M_{i}\right) \cong M_{i}$ for all $i$. This means that

$$
M=\bigcup_{i=0}^{\infty} M_{i} \cong \bigcup_{i=0}^{\infty} Y \circ F\left(M_{i}\right)=Y \circ F\left(\bigcup_{i=0}^{\infty} M_{i}\right)=Y \circ F(M)
$$

as tensoring commutes with direct limits ([R, Corollary 2.10]).

Given any $M, M^{\prime} \in \mathscr{O}_{J}(\lambda)$, we have a canonical isomorphism,

$$
\operatorname{Hom}_{U_{q}(\mathfrak{g})}\left(M, M^{\prime}\right) \cong \operatorname{Hom}_{\mathfrak{p}}\left(F(M), F\left(M^{\prime}\right)\right)=\operatorname{Hom}_{\mathfrak{p}^{\prime}}\left(M^{f},\left(M^{\prime}\right)^{f}\right)
$$

as $M$ and $M^{\prime}$ are generated by their finite parts. Similarly, for any $N, N^{\prime} \in \mathscr{O}^{J}(\lambda)$, we have a canonical isomorphism

$$
\operatorname{Hom}_{\mathfrak{p}},\left(N, N^{\prime}\right) \cong \operatorname{Hom}_{U_{q}(\mathfrak{g})}\left(Y(N), Y\left(N^{\prime}\right)\right) .
$$


We have shown that the compositions of functors $F \circ Y$ and $Y \circ F$ are naturally equivalent to the identity functors $I_{\sigma_{J}(\lambda)}$ and $I_{\sigma^{J}(\lambda)}$ respectively. Hence the categories $\theta_{J}(\lambda)$ and $\mathscr{O}^{J}(\lambda)$ are equivalent.

For completeness we note the following consequence of Theorem 6.5 .

COROllaRY 6.6. Let $N \in \mathscr{O}_{J}(\lambda)$. Then $N \cong U_{q}(\mathfrak{g}) \otimes_{\mathfrak{p}^{J}} N^{f}$ as $U_{q}(\mathfrak{g})$-module.

\section{References}

[Be] J. Beck, 'Braid group action and quantum affine algebras', Comm. Math. Phys. 165 (1994), 555-568.

[BK] J. Beck and V. G. Kac, 'Finite-dimensional representations of quantum affine algebras at roots of unity', J. Amer. Math. Soc. 9 (1996), 391-423.

[CFKM] B. Cox, V. M. Futorny, S.-J. Kang and D. J. Melville, 'Quantum deformations of imaginary Verma modules', Proc. London Math. Soc. 74 (1997), 52-80.

[CFM] B. Cox, V. M. Futorny and D. J. Melville, 'Categories of nonstandard highest weight modules for affine Lie algebras', Math. Z. 221 (1996), 193-209.

[FO] M. Fabbri and F. Okoh, 'Representations of quantum Heisenberg algebras', Canad. J. Math. 46 (1994), 920-929.

[Ful] V. M. Futorny, 'Root systems, representations and geometries', Ac. Sci. Ukrain. Math. 8 (1990), 30-39.

[Fu2] _ _ The parabolic subsets of root systems and corresponding representations of affine Lie algebras', Contemp. Math. 131 (1992), 45-52.

[Fu3] _ 'Irreducible non-dense $A_{1}^{(1)}$-modules', Pac. J. Math. 172 (1996), 83-99.

[Fu4] - Representations of affine Lie algebras, Queen's Papers in Pure and Appl. Math. 106 (Queen's University, Kingston, 1997).

[FGM] V. M. Futorny, A. N. Grishkov and D. J. Melville, 'Verma-type modules for quantum affine Lie algebras', preprint 1998.

[JK1] H. P. Jakobsen and V. G. Kac, A new class of unitarizable highest weight representations of infinite dimensional Lie algebras, Lecture Notes in Phys. 226 (Springer, Berlin, 1985) pp. 1-20.

[JK2] _ 'A new class of unitarizable highest weight representations of infinite dimensional Lie algebras II', J. Funct. Anal. 82 (1989), 69-90.

[KT] S. M. Khoroshkin and V. N. Tolstoy, 'On Drinfeld's realization of quantum affine algebras', J. Geom. Phys. 11 (1993), 445-452.

[RW] A. Rocha-Caridi and N. Wallach, 'Projective modules over graded Lie algebras. I', Math. Z. 180 (1982), 151-177.

[R] J. J. Rotman, An introduction to homological algebra (Academic Press, New York, 1979).

Instituto de Matematica

Universidade do Sao Paulo

Sao Paulo

Brasil

e-mail: futorny@ime.usp.br
Department of Mathematics

St. Lawrence University

Canton, New York 13617

USA

e-mail: dmel@music.stlawu.edu 